OPEN ACCESS

Edited by:

Manousos A. Klados, Aston University, United Kingdom

Reviewed by:

Feng Liu,

Tianjin Medical University General Hospital, China

Zsigmond Tamás Kincses, University of Szeged, Hungary

*Correspondence: $\mathrm{Bin} \mathrm{Hu}$

bh@lzu.edu.cn

Lingjiang Li

Ilj2920@163.com

Received: 17 August 2017 Accepted: 30 October 2017 Published: 05 December 2017

Citation:

Zhang Z, Liao M, Yao Z, Hu B, Xie Y, Zheng $W$, Hu T, Zhao Y, Yang F, Zhang Y, Su L, Li L, Gutknecht J and Majoe D (2017) Frequency-Specific

Functional Connectivity Density as an Effective Biomarker for Adolescent Generalized Anxiety Disorder. Front. Hum. Neurosci. 11:549. doi: 10.3389/fnhum.2017.00549

\section{Frequency-Specific Functional Connectivity Density as an Effective Biomarker for Adolescent Generalized Anxiety Disorder}

\begin{abstract}
Zhe Zhang', Mei Liao 2,3, Zhijun Yao', Bin Hu',4*, Yuanwei Xie', Weihao Zheng ${ }^{1}$, Tao Hu', Yu Zhao', Fan Yang ${ }^{5}$, Yan Zhang ${ }^{2,3}$, Linyan Su ${ }^{2,3}$, Lingjiang Li2,3*, Jürg Gutknecht ${ }^{6}$ and Dennis Majoe ${ }^{6}$

' Gansu Provincial Key Laboratory of Wearable Computing, School of Information Science and Engineering, Lanzhou University, Lanzhou, China, ${ }^{2}$ Mental Health Institute of the Second Xiangya Hospital, Central South University, Changsha, China, ${ }^{3}$ The China National Clinical Research Center for Mental Health Disorders, National Technology Institute of Psychiatry, Key Laboratory of Psychiatry and Mental Health of Hunan Province, Changsha, China, ${ }^{4}$ CAS Center for Excellence in Brain Science and Intelligence Technology, Shanghai Institutes for Biological Sciences, Chinese Academy of Sciences, Shanghai, China, ${ }^{5}$ Guangdong Mental Health Center, Guangdong General Hospital, Guangzhou, China, ${ }^{6}$ Computer Systems Institute, ETH Zürich, Zürich, Switzerland
\end{abstract}

Several neuropsychiatric diseases have been found to influence the frequency-specific spontaneous functional brain organization (SFBO) in resting state, demonstrating that the abnormal brain activities of different frequency bands are associated with various physiological and psychological dysfunctions. However, little is known about the frequency specificities of SFBO in adolescent generalized anxiety disorder (GAD). Here, a novel complete ensemble empirical mode decomposition with adaptive noise method was applied to decompose the time series of each voxel across all participants (31 adolescent patients with GAD and 28 matched healthy controls; HCs) into four frequency-specific bands with distinct intrinsic oscillation. The functional connectivity density (FCD) of different scales (short-range and long-range) was calculated to quantify the SFBO changes related to GAD within each above frequency-specific band and the conventional frequency band $(0.01-0.08 \mathrm{~Hz})$. Support vector machine classifier was further used to examine the discriminative ability of the frequencyspecific FCD values. The results showed that adolescent GAD patients exhibited abnormal alterations of both short-range and long-range FCD (S-FCD and L-FCD) in widespread brain regions across three frequency-specific bands. Positive correlation between the State Anxiety Inventory (SAl) score and increased L-FCD in the fusiform gyrus in the conventional frequency band was found in adolescents with GAD. Both S-FCD and L-FCD in the insula in the lower frequency band $(0.02-0.036 \mathrm{~Hz})$ had the highest classification performance compared to all other brain regions with inter-group difference. Furthermore, a satisfactory classification performance was achieved by combining the discrepant S-FCD and L-FCD values in all frequency bands, with the area under the curve (AUC) value of 0.9414 and the corresponding sensitivity, specificity, and accuracy of $87.15,92.92$, and $89.83 \%$, respectively. This study indicates that the 
alterations of SFBO in adolescent GAD are frequency dependence and the frequencyspecific FCD can potentially serve as a valuable biomarker in discriminating GAD patients from $\mathrm{HCs}$. These findings may provide new insights into the pathophysiological mechanisms of adolescent GAD.

Keywords: adolescent generalized anxiety disorder, frequency-specific, functional connectivity density, CEEMDAN, classification

\section{INTRODUCTION}

Generalized anxiety disorder (GAD), as a common subtype of anxiety disorder, is a prevalent psychiatric disease which often occurs in adolescence or early adulthood, characterized by frequently excessive as well as uncontrollable fear and worry about a variety of things such as school life, social work, and individual ability (Beesdo et al., 2010). Moreover, GAD patients are often easily fatigued, restless, and difficult to concentrate or blank in mind with symptoms of muscle tension, thus contributing to distress and functional impairment, which severely affects patients' quality of life and to some extent increases social and economic burden (Kessler et al., 2005; Hoffman et al., 2008). To make things worse, GAD patients spend more time on treatment due to lower disease recognition rate and higher degree of differentiation compared with other types of anxiety disorder (Chavira et al., 2004). During the past decades, although a vast number of studies have focused on exploring GAD as well as adolescents with GAD, unfortunately, the pathophysiological mechanisms of GAD still remain poorly understood.

Resting-state functional connectivity (FC) that measures the temporal coherence of blood oxygen level-dependent (BOLD) signals between two spatially distinct regions has been widely applied to explore the spontaneous functional brain organization (SFBO) of GAD. Previous GAD studies have focused largely on amygdala-based connectivity and showed aberrant amygdala connectivity with several brain regions including prefrontal cortex (Monk et al., 2008; Etkin et al., 2009), anterior cingulate cortices (Etkin et al., 2010; Blair et al., 2012), insula (Hamm et al., 2014), and limbic system (Roy et al., 2013; Liu et al., 2015c), which indicates that the amygdala may play an important role in functional neural circuitry underlying the progression of GAD symptomatology (Hilbert et al., 2014; Makovac et al., 2016). Recently, a few studies have extended the investigation of abnormal FC of GAD patients to more brain regions. The alteration of FC in GAD patients has been found between prefrontal and limbic brain regions that may be related to emotional regulation deficits in GAD (Wang W. et al., 2016). However, all of the above-mentioned resting-state FC studies of GAD have mainly focused on BOLD signals within a broad frequency band $(0.01-0.08 \mathrm{~Hz})$, so some meaningful information related to specific frequency bands underling GAD may be missing.

It is widely accepted that human brain is a complex biological system in which a considerable number of oscillatory waves work together. With regard to the signals within different frequency bands, they may come from distinct oscillators with specific neural processes (Buzsa and Draguhn, 2004; Athanasiou et al., 2016) and physiological functions (Knyazev, 2007). Therefore, subdivision of the frequency band $(0.01-0.08 \mathrm{~Hz})$ may be an efficient approach in exploring specific functional activities in both healthy controls (HCs) and patients with mental disorders. For example, by carrying out a wholebrain amplitude low-frequency fluctuation (ALFF) analysis, a resting-state fMRI study observed the spontaneous activity at four different frequency bands (slow-5: $0.01-0.027 \mathrm{~Hz}$, slow-4: $0.027-0.073 \mathrm{~Hz}$, slow-3: $0.073-0.198 \mathrm{~Hz}$, and slow-2: $0.198-0.25 \mathrm{~Hz}$ ) in healthy human brain. Specifically, the activities in slow-5 were more active on cortical structures, whereas activities in slow-4 decreased in the subcortical structures, suggesting that the spontaneous brain activities were frequencydependent (Zuo et al., 2010). Additionally, frequency-specific changes of SFBO have also been found in several psychiatric and neurological diseases, such as depression (Wang L. et al., 2016), social anxiety disorder (Zhang et al., 2015), epilepsy (Wang et al., 2015), Parkinson's disease (Qian et al., 2016), mild cognitive impairment (Han et al., 2011), internet gaming disorder (Lin et al., 2015), migraine (Farago et al., 2017), and Wilson's disease ( $\mathrm{Hu}$ et al., 2017). To date, little is known about the frequency specificities of SFBO in adolescent GAD.

Moreover, the majority of research on GAD has revealed aberrant FC concentrated on seed-based analyses or predefined networks, which may neglect some unpredictable findings, because neither seed-based analyses nor predefined networks can effectively measure the FCs of the whole-brain. Recently, functional connectivity density (FCD) mapping, a novel voxelwise FC method, has been developed to detect SFBO by measuring the local centrality of every voxel in the wholebrain connection (Tomasi and Volkow, 2010; Tomasi et al., 2016). Unlike the seed-based FC approach, FCD is a datadriven method, the requirement of no priori-knowledge making it more proper for exploratory analyses (Tomasi et al., 2016). In general, FCD reflects the complexity of the connectome as a whole and can fully exhibit the altered cortical and subcortical functional hubs underlying SFBO. According to the neighboring relationship between two brain voxels, FCD can be further divided into short-range FCD (S-FCD, the FC in local regions) and long-range FCD (L-FCD, the subtraction of FC between in the whole-brain and a local region) (Tomasi and Volkow, 2012), which can be served as indicators to explore abnormal functional organization in blind participants (Qin et al., 2015), post-traumatic stress disorder (Zhang et al., 2016), male heavy drinkers (Shokri-Kojori et al., 2016), irritable bowel syndrome (Weng et al., 2016), generalized tonic-clonic seizures (Zhu et al., 
2016), and adolescents with pure conduct disorder ( $\mathrm{Lu}$ et al., 2017).

So far, the mechanism of GAD-related alterations in frequency-specific FCD remains unclear. The present study aims to investigate how GAD affects the S-FCD and L-FCD in adolescents across different frequency bands. The complete ensemble empirical mode decomposition with adaptive noise (CEEMDAN) was applied to decompose the BOLD signals into four different frequency bands, and the S-FCD and L-FCD in each frequency band were calculated in further analysis. Additionally, we also aim to examine whether the frequencyspecific FCD could help to discriminate patients with GAD from HCs. Specifically, support vector machine (SVM) with linear kernel was performed in discrimination between the patients and controls. Previous findings have revealed whole-brain FC had a good diagnostic potential for anxiety disorder (Liu et al., 2015a). Moreover, FC changes would reflect alterations in GAD symptomatology and can be as a treatment-relevant biomarker of symptom expression (Makovac et al., 2016). Therefore, we hypothesized that frequency-specific FCD could be used as a potential biomarker to distinguish patients from controls.

\section{MATERIALS AND METHODS}

\section{Participants}

A total of 59 participants were recruited from local high schools in Hunan Province, including 31 GAD patients and 28 age- and sex- matched HCs. All the participants were enrolled via advertisements and school notice, which have been demonstrated in our previous study (Liao et al., 2013, 2014). Firstly, 1885 subjects finished the Screen for Child Anxiety Related Emotional Disorders (SCARED) which is a 41-item selfreport questionnaire. The SCARED turns out to be a reliable and valid screening tool for childhood anxiety disorder, and it has an optimal total cutoff point score of 25 to separate children who are with anxiety disorders from those without ( $\mathrm{Su}$ et al., 2008). Among 1885 subjects, 508 subjects' SCARED scores were higher than or equal to 25 , while 1377 subjects' SCARED scores were lower than 25 . Then, 673 subjects (508 SCARED scores $\geq 25 ; 165$ SCARED scores $<25$ ) were investigated by the same trained clinician and diagnosed using DSM-IV criteria and the Schedule for Affective Disorders and Schizophrenia for School Age Children-Present and Lifetime (K-SADS-PL) version (Lauth et al., 2010). To assess clinical activity, all participants performed the State-Trait Anxiety Inventory (STAI) (Spielberger et al., 1970), the Penn State Worry Questionnaire (PSWQ) (Meyer et al., 1990), and the Beck Depression Inventory (BDI) (Beck and Beamesderfer, 1974) before the fMRI scan. STAI is a self-administered evaluation system to assess anxiety severity, such as trait anxiety and state anxiety. PSWQ, consisting of 16 self-report items, is considered as a reliable scale to evaluate worry frequency and intensity, and with good testretest reliability and enabling to distinguish GAD patients from patients with other anxiety disorders. BDI, a rating inventory containing 21 self-report items, can assess characteristic attitudes and depression symptoms, and shows high internal consistency in psychiatric populations and non-psychiatric, respectively. Specifically, participants with current first episode GAD without co-morbidity disorders and HCs without mental disorders and physical disease were included, whereas participants with history of seizures, neurological abnormalities, head trauma or unconsciousness, physical disease, and use of psychoactive substances were excluded. All participants were ensured to be non-medicated, right-handed, and voluntary. Each participant and one of his or her legal guardians were given written informed consent before the study. This study was approved by the local medical ethics committee at the Second Xiangya Hospital of Central South University. The demographic and neuropsychological characteristics of all the participants are detailed in Table 1.

\section{Data Acquisition and Preprocessing}

All data were collected using a 3.0 Tesla Philips scanner equipped with a SENSE-8 channel head coil at the Second Xiangya Hospital of Central South University, Hunan, China. Before the scan, participants were instructed to lie relaxed, keep eyes closed, stay awake, think of nothing in particular, and move as little as possible during scanning. Head motion and scanner noise were controlled by foam paddings and earplugs. After fMRI scan, each participant was given a questionnaire to ensure whether he/she had fallen asleep or opened eyes during the time of scanning and no participant's data was excluded. High-resolution T1-weighted anatomical images were acquired for registration in the sagittal orientation using a magnetization-prepared rapid gradient-echo sequence (repetition time $=7.5 \mathrm{~ms}$, echo time $=3.7 \mathrm{~ms}$, slice thickness $=1 \mathrm{~mm}$, field of view $=256 \mathrm{~mm} \times 256 \mathrm{~mm}$, flip angle $=8^{\circ}$, and 180 transverse slices covered the whole brain). Resting-state fMRI data were acquired using a singleshot, gradient-recalled echo planar imaging sequence (repetition time $=3000 \mathrm{~ms}$, echo time $=30 \mathrm{~ms}$, slice thickness $=4 \mathrm{~mm}$, slice gap $=0.38 \mathrm{~mm}$, field of view $=240 \mathrm{~mm} \times 240 \mathrm{~mm}$, in-plane matrix $=64 \times 64$, flip angle $=90^{\circ}$, and 36 slices covered the

TABLE 1 | Demographics and neuropsychological characteristics of the participants.

\begin{tabular}{lccc}
\hline $\begin{array}{l}\text { Characteristics } \\
\text { Characteristics }\end{array}$ & $\begin{array}{c}\text { GAD }(\boldsymbol{n}=\mathbf{3 1}) \\
(\text { Mean } \pm \text { SD) }\end{array}$ & $\begin{array}{c}\text { HC }(\boldsymbol{n}=\mathbf{2 8}) \\
(\text { Mea } \mathbf{n} \pm \mathbf{S})\end{array}$ & $\boldsymbol{P}$-value \\
\hline Age (years) & $16.90 \pm 0.12$ & $16.46 \pm 0.18$ & $0.44^{\mathrm{a}}$ \\
Handedness (right/left) & $31 / 0$ & $28 / 0$ & $0.99^{\mathrm{b}}$ \\
Gender (males/females) & $14 / 17$ & $16 / 12$ & $0.36^{\mathrm{b}}$ \\
IQ & $102.7 \pm 7.86$ & $105.4 \pm 9.20$ & $0.19^{\mathrm{a}}$ \\
STAI & & & \\
$\quad$ State & $43.48 \pm 8.51$ & $39.86 \pm 7.87$ & $0.02^{\mathrm{a}}$ \\
$\quad$ Trait & $52.34 \pm 7.63$ & $44.25 \pm 9.18$ & $0.00^{\mathrm{a}}$ \\
BDI & $8.36 \pm 4.60$ & $6.12 \pm 5.21$ & $0.10^{\mathrm{a}}$ \\
PSWQ & $55.16 \pm 9.76$ & $38.79 \pm 11.26$ & $0.00^{\mathrm{a}}$ \\
\hline
\end{tabular}

Values represented mean $\pm S D$. $S D$, standard deviation; $I Q$, intelligence quotient; STAI, State-trait Anxiety Inventory; BDI, Beck Depression Inventory; PSWQ, Penn State Worry Questionnaire; GAD, generalized anxiety disorder; HC, healthy control. aThe $P$-value were obtained by two-sample t-tests. ${ }^{b}$ The $P$-value were obtained by Chi square test. 
whole brain) to measure 200 functional volumes for each subject in a total scan time of 600 seconds.

The data preprocessing was performed using DPARSF ${ }^{1}$ based on SPM12. The main preprocessing steps included: (1) the first 10 functional images were discarded to stabilize the signal and adapt to inherent scanner noise. (2) The remaining functional images were corrected by slice-timing calibrating and head motion realigning. Specifically, they were first corrected differences in within-scan acquisition time among slices and then were realigned to the first volume for the purpose of correcting inter-scan head motion. Any data affected by head motion (maximal motion between volumes in each direction is greater than $3 \mathrm{~mm}$, and rotation in each axis is greater than $3^{\circ}$ ) were discarded. We also calculated the mean framewise displacement (FD) of each participant on the basis of realignment parameters and the FD of each participant was less than $0.3 \mathrm{~mm}$. There was no significant difference in $\mathrm{FD}$ (GAD: $0.12 \pm 0.011$; HC: $0.13 \pm 0.013 ; P=0.47$ ) between two groups using two-sample $t$-test. (3) Functional images were spatially normalized to a standard three-dimensional space with the Montreal Neurological Institute (MNI) template and resampled to a resolution of $3 \mathrm{~mm} \times 3 \mathrm{~mm} \times 3 \mathrm{~mm}$. To be more exact, a rigid-body transformation was used to co-register the individuals' the high resolution structural images to the mean functional images. Then, all the co-registered images were segmented into gray matter, white matter and cerebrospinal fluid in MNI space by using "New Segment+DARTEL" in DPARSF. We applied the DARTEL procedure (Ashburner, 2007) to generate a study-specific template. (4) Linearly detrending and low-pass filtering $(0-0.08 \mathrm{~Hz})$ were performed to discard signal drifts caused by scanner instability or other sources. Particularly, we selected low-pass filtering rather than bandpass filtering $(0.01-0.08 \mathrm{~Hz})$ because previous studies have investigated brain activity within the frequency band of $0-0.01 \mathrm{~Hz}$ (Lin et al., 2015; Wang et al., 2015). (5) Nuisance covariates including head motion parameters, global mean signal, white matter signal and cerebrospinal signal were regressed out from the BOLD signals. Here, we removed global mean signals because, according to previous studies, the global signal removal can reduce physiological noise and improve the reliability of restingstate fMRI (Yan et al., 2013).

\section{Definition of Frequency of Interest (FOI)}

After data preprocessing, a CEEMDAN method was applied to decompose the BOLD signals of each voxel into a finite set of intrinsic oscillatory components called intrinsic mode functions (IMFs), and each IMF occupies a unique frequency band (Colominas et al., 2014). CEEMDAN was developed from empirical mode decomposition (EMD) (Huang et al., 1998), which automatically decomposes non-stationary signals in a data-driven manner without any rigid predefined band-pass filter. However, the disadvantage of EMD would easily result in the "mode mixing" phenomenon, which always produces oscillations with very disparate frequencies in one IMF, or

\footnotetext{
${ }^{1}$ http://www.restfmri.net

${ }^{2}$ http://www.fil.ion.ucl.ac.uk/spm
}

oscillations with similar frequencies in different IMFs. Also, the IMFs of EMD might contain some residual noise. CEEMDAN is a significant improvement on EMD, which are able to solve the problem of mode mixing and eliminate the residue noise in each IMF. For a given time series $x(t)$, the CEEMDAN aims to extract a set of IMFs $\left(\operatorname{IMF}_{i}(t), i=1,2, \cdots, K\right)$ and a monotonic residue signal $r(t)$, so that $x(t)=\sum_{i=1}^{k} I M F_{i}(t)+r(t)$. In this equation, the $t, i, K$ denote the length of scanning time, the order of IMF, the number of IMF, respectively. CEEMDAN adopts an iterative method known as the sifting algorithm based on EMD to extract IMFs. The algorithm includes following steps: (1) Obtain the first residue by EMD. (2) Calculate the first IMF by subtracting the first residue from the raw signal. (3) Estimate the second residue and define the second IMF. (4) Repeat these steps until the last IMF is obtained. The detailed algorithm is presented in literature (Colominas et al., 2014). As a result of CEEMDAN, each IMF component occupies a distinctive frequency band: the first IMF occupies the highest frequency band, while the last IMF occupies the lowest frequency band, with the remaining ones in between, respectively.

After decomposition, the Hilbert weighted frequency (HWF) was applied to reflect the mean oscillation frequency of an IMF through instantaneous spectra information about amplitude and phase (Xie and Wang, 2006; Song et al., 2014). A brief computing step of HWF includes: (1) Compute Hilbert transform for each IMF. (2) Calculate the corresponding analytical signal. (3) Compute the HWF of each IMF with all data points. The detailed computing steps can be found in literature (Qian et al., 2016). Then we calculated all HWFs corresponding to IMFs in the whole brain gray matter across all participants and obtained the HWF distribution histograms in each group. In order to identify isolated frequency bands and lower the influence of extreme values, we used each corresponding HWF distribution component in $95 \%$ confidence intervals to derive a frequency of interest (FOI). Hence, the frequency band for each FOI would not overlap and can be considered independent from each other. To ensure the consistency of FOI between two groups, we chose maximum of lower limit and minimum of upper limit within each group frequency bands identified by $95 \%$ confidence interval to be lower and higher limiting value of final FOI, respectively. Besides, for comparison purposes, conventional frequency band from 0.01 to $0.08 \mathrm{~Hz}$ was selected as normal frequency of interest (FOI-N).

\section{Frequency-Specific FCD Mapping}

The FCD mapping was generated by computing the number of functional connections of each voxel with the rest voxels in the whole-brain (Tomasi and Volkow, 2010). Usually, the FCD was divided, according to the functional connection properties (for example the length of connectivity), to short-range FCD (S-FCD) and long-range FCD (L-FCD). The S-FCD reflects the FC between voxels within a local cluster (intraregional), while L-FCD reflects the FC between a voxel within a local and the other without the local cluster (interregional) (Tomasi and Volkow, 2012). For a given voxel $x_{0}$, its local cluster was determined by using a fast searching algorithm. Mathematically, the S-FCD value of a voxel $x_{0}$ is computed as the numbers of 


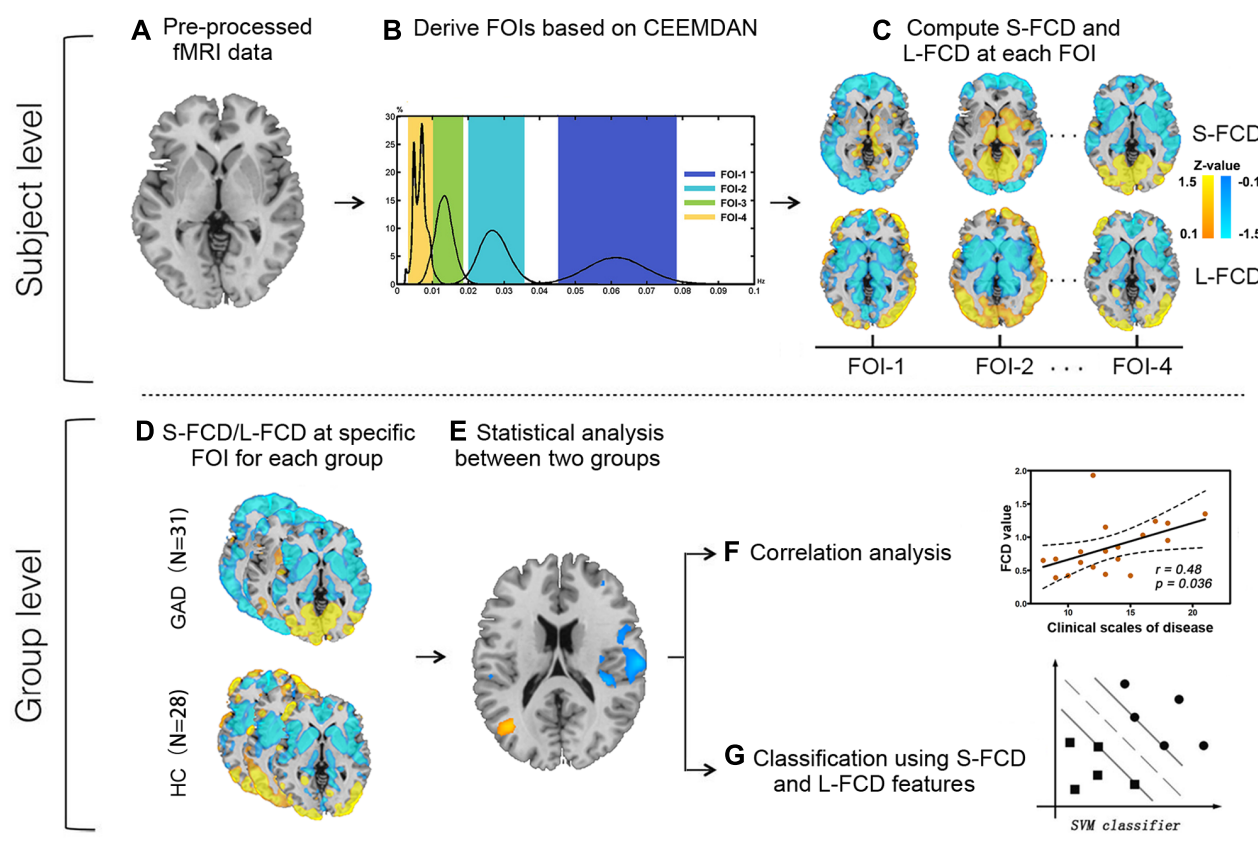

FIGURE 1 | Flow diagram illustrating the proposed research framework. CEEMDAN, complete ensemble empirical mode decomposition with adaptive noise; S-FCD, short-range functional connectivity density; L-FCD, long-range functional connectivity density; FOI, frequency of interest; GAD, generalized anxiety disorder; $\mathrm{HC}$, healthy control.

functional connections above a correlation threshold between voxel $x_{0}$ and the other voxels belong to the local cluster. This calculation is repeated for all voxels within whole-brain gray matter. Here, Pearson correlation analysis was introduced to evaluate the functional connection between a pair of voxels, and a correlation threshold above 0.6 was considered functionally connected, which proposed to be the optimal threshold for calculating FCD in previous studies (Zhang et al., 2016; Zhu et al., 2016). Similarly, the L-FCD equated to the numbers of functional connections between the voxel $x_{0}$ within a local cluster and all other voxels without the local cluster in the whole-brain gray matter. The detailed computing procedure about S-FCD and L-FCD can be found in Tomasi and Volkow (2012).

The S-FCD and L-FCD within each predefined FOI of all participants were standardized by converting into $z$-scores. Then, the $z$-scored FCD maps were spatially smoothed with a full-width at half-maximum (FWHM) Gaussian kernel of $6 \mathrm{~mm}$ to minimize the differences in the functional anatomy of the brain across participants.

\section{Statistical and Classification Analyses}

Two-sample $t$-tests and chi-square tests were implemented to investigate the group differences in continuous and categorical variables between the GAD patients and HCs by using SPSS21, respectively. Voxel-based comparisons of FCD mappings within the whole-brain gray matter mask were performed using twosample $t$-tests with the DPABI software ${ }^{3}$. The resulting statistical maps were set at a corrected $P<0.05$, with a cluster size of at

${ }^{3} \mathrm{http}: / / \mathrm{rfmri.org} / \mathrm{dpabi}$ least 220 voxels above an uncorrected $P<0.001$, with estimated FWHM (14.22, 15.91, 15.69), performed by the AlphaSim program. Pearson correlation was conducted between FCD value and clinical questionnaires (STAI, BDI, and PSWQ) score of GAD patients in brain regions with significant group differences, with age and gender as covariates to be regressed out. Values with $P<0.05$ (FDR corrected) were considered to indicate statistical significance.

We further investigated whether the frequency-specific S-FCD/L-FCD with significant inter-group differences could discriminate the GAD patients from the HCs by means of the SVM classifier. SVM is a popular classifier and well-performed supervised learning model which works by projecting the lowdimensional non-separable data to high-dimensional separable data (Cortes and Vapnik, 1995). SVM with a linear kernel was used to reduce the risk of overfitting the training data and directly extract the feature weights (Pereira et al., 2009). We utilized "leave-one-out" cross-validation method to provide an unbiased estimation of the performance of aforementioned features. Specifically, in each cross-validation experiment, one participant was selected as the test subject, and the rest 58 participants were viewed as the training set. The intergroup differences in frequency-specific S-FCD/L-FCD within the training set were detected by conducting two-sample t-test and the mean S-FCD/L-FCD values of the voxels in the intergroup difference clusters were also calculated as classification features. We repeated this procedure 59 times. Moreover, the receiver operating characteristic (ROC) curves and the area under the curves (AUC) were used to evaluate whether FCD with significant between-group differences could be utilized 


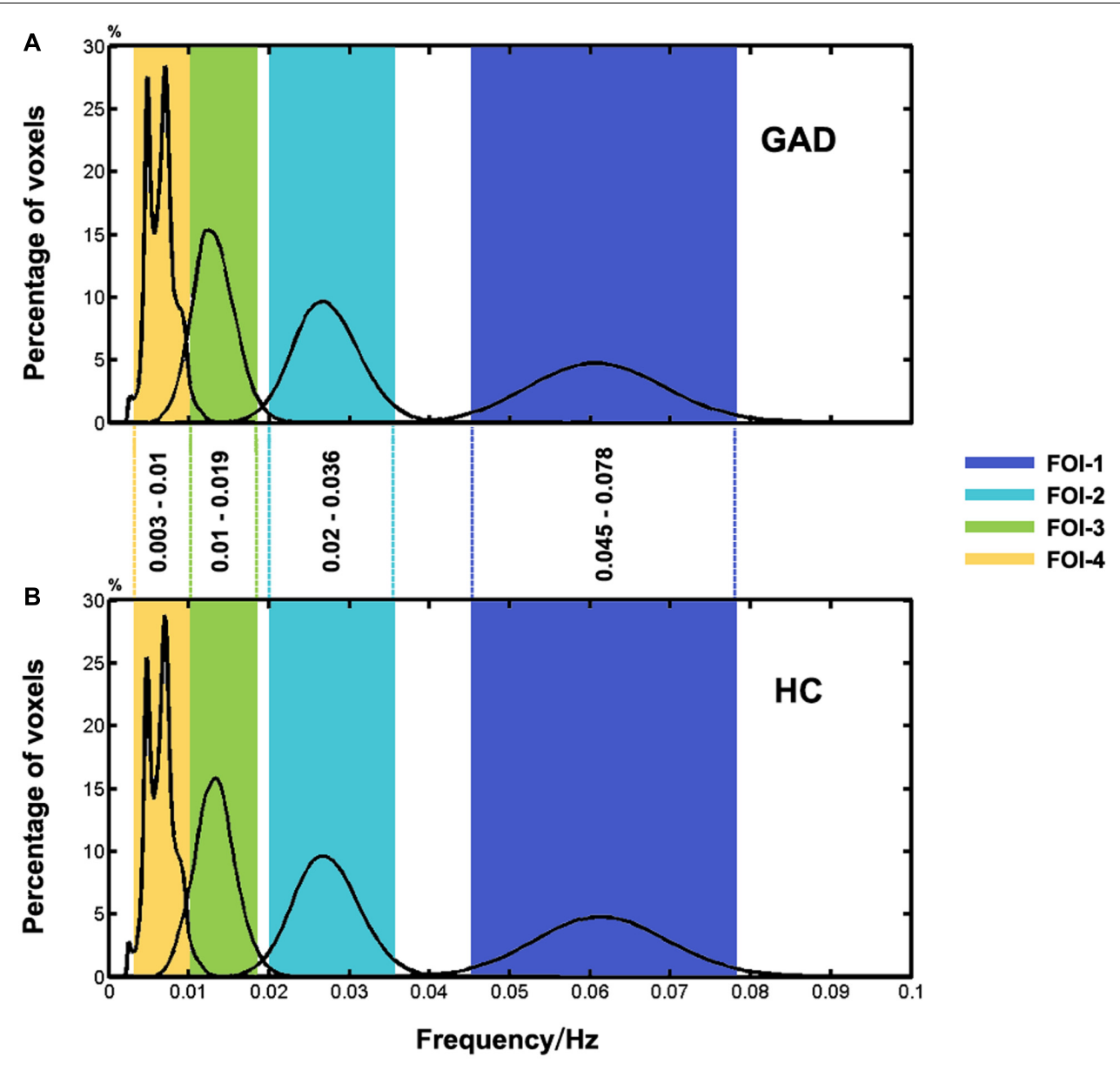

FIGURE 2 | Histogram of frequency distribution between two groups. The histograms of the HWF distributions show the first four intrinsic mode functions (IMFs) of each voxel in the whole-brain gray matter across all participants in each group by using the CEEMDAN approach. Each of the sub-histograms in corresponding IMFs displays amount of the whole-brain gray matter voxels within the GAD (A) and HC (B) group, respectively. Vertical axis represents the percentage of the number of voxels with HWF equal to the frequency on the horizontal axis in the whole-brain gray matter. The rectangular bar with different colors represents the frequency bands of the final selected FOls. Colors assigned in the sequence of blue, cyan, green, and yellow represent FOl-1 (0.045-0.078 Hz), FOI-2 (0.02-0.036 Hz), FOI-3 $(0.01-0.019 \mathrm{~Hz})$, and FOI-4 $(0.003-0.01 \mathrm{~Hz})$, respectively.

as markers to discriminate the GAD patients from the HCs. All the above classification analysis were conducted by the LIBSVM 3.22 matlab toolbox ${ }^{4}$. An overview of the proposed classification procedure with frequency-specific FCD framework was summarized in Figure 1.

\section{RESULTS}

\section{Frequency Distribution of IMF in GAD and $\mathrm{HC}$}

As shown in Figure 2, four FOIs intervals $(0.045-0.078 \mathrm{~Hz}, 0.02-$ $0.036 \mathrm{~Hz}, 0.01-0.019 \mathrm{~Hz}$, and $0.003-0.01 \mathrm{~Hz}$ ) were finally selected to ensure the consistency of frequency bands between the two groups. For simplicity, we numbered them from FOI-1 to FOI-4, where FOI-1 was the highest frequency interval and FOI-4 was

${ }^{4}$ http://www.csie.ntu.edu.tw/ cjlin/libsvm/ the lowest. We named these four intervals together as FOI-S (including FOI-1 to FOI-4) when compared with FOI-N.

\section{Frequency-Specific FCD Alterations in GAD}

We observed between-group differences of the S-FCD and L-FCD in conventional frequency band FOI-N $(P<0.05$, AlphaSim corrected, with a cluster size of 220 voxels). Compared to the HC, S-FCD of GAD patients significantly increased in the left middle temporal gyrus (MTG), but decreased in the right postcentral gyrus (PosCG) and left middle frontal gyrus (MFG). Meanwhile, L-FCD in GAD patients increased in the left fusiform gyrus (FG), but decreased in the right precentral gyrus (PreCG) and right inferior parietal lobule (IPL) (see Table 2 and Figure 3).

Significant between-group differences of the S-FCD and L-FCD were also found in three frequency-specific bands. Compared to the HC, GAD patients showed significant 
TABLE 2 | Brain areas showing significant differences in FCD between patients with GAD and HCs in FOI-N (0.01-0.08 Hz).

\begin{tabular}{|c|c|c|c|c|c|c|}
\hline \multirow[t]{2}{*}{ Anatomical regions } & \multirow[t]{2}{*}{ BA } & \multicolumn{3}{|c|}{ MNI coordinates } & \multirow[t]{2}{*}{ Cluster size } & \multirow[t]{2}{*}{ Maximal $t$-value } \\
\hline & & $x$ & $Y$ & $Z$ & & \\
\hline \multicolumn{7}{|l|}{ S-FCD } \\
\hline \multicolumn{7}{|l|}{$\mathrm{GAD}<\mathrm{NC}$} \\
\hline Right postcentral gyrus (PosCG) & 4 & 63 & -15 & 18 & 599 & -3.99 \\
\hline Left middle frontal gyrus (MFG) & $6 / 32$ & -15 & -6 & 63 & 265 & -5.06 \\
\hline \multicolumn{7}{|l|}{ GAD > NC } \\
\hline Left middle temporal gyrus (MTG) & 39 & -45 & -72 & 21 & 254 & 3.12 \\
\hline \multicolumn{7}{|l|}{ L-FCD } \\
\hline \multicolumn{7}{|l|}{$\mathrm{GAD}<\mathrm{NC}$} \\
\hline Right precentral gyrus (PreCG) & 4 & 45 & -18 & 39 & 364 & -3.56 \\
\hline Right inferior parietal lobule (IPL) & 40 & 36 & -42 & 57 & 252 & -4.52 \\
\hline \multicolumn{7}{|l|}{ GAD > NC } \\
\hline Left fusiform gyrus (FG) & 20 & -51 & -6 & -27 & 246 & 4.40 \\
\hline
\end{tabular}

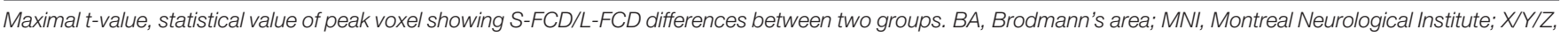
coordinates of peak locations in the MNI space. $P<0.05$, corrected for AlphaSim.

frequency-specific S-FCD and L-FCD alterations $(P<0.05$, AlphaSim corrected, with a cluster size of 220 voxels, see Table 3 and Figure 4). Specifically, S-FCD of GAD patients in the left superior frontal gyrus (SFG) (FOI-1) and right FG (FOI-3) significantly increased, but decreased in the right IPL (FOI-1) and right insula (INS) (FOI-2). Further, L-FCD of GAD patients decreased in the right IPL (FOI-1), right PosCG (FOI-2), and right INS (FOI-2 and FOI-3). No significant between-group difference was found in frequency band FOI-4.

\section{A S-FCD: GAD > HC FOI-N $(0.01-0.08 \mathrm{~Hz})$}

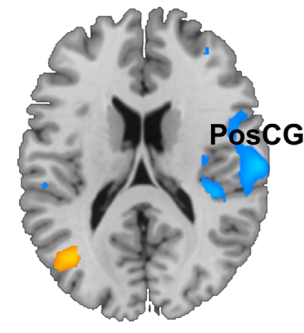

$Z=18$

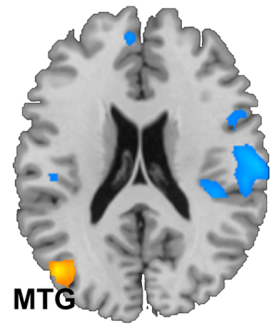

$Z=21$

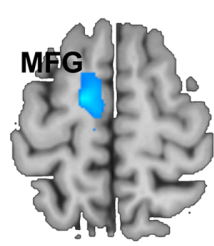

$Z=63$

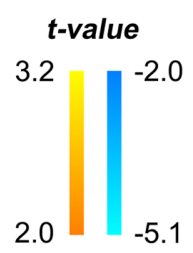

B L-FCD: GAD > HC FOI-N $(0.01-0.08 \mathrm{~Hz})$

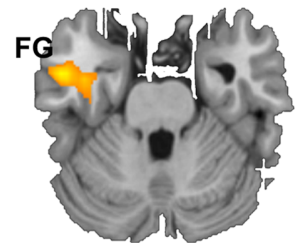

$Z=-27$

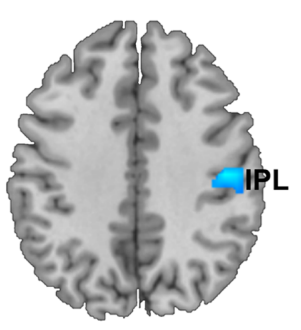

$Z=39$

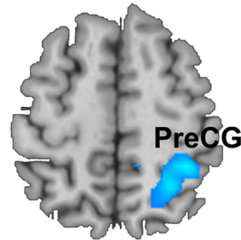

$Z=57$

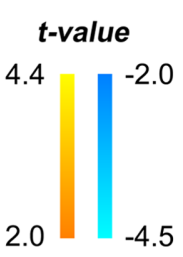

FIGURE 3 | Statistical differences of FCD between two groups in the conventional frequency band FOI-N $(0.01-0.08 \mathrm{~Hz})$. (A) Statistical differences of S-FCD between two groups. (B) Statistical differences of L-FCD between two groups. The color bar indicates the $t$ values, which represent increased (warm) and decreased (cool) S-FCD and L-FCD in the GAD patients compared with HCs. Two sample $t$-test, significant level was set at $P<0.05$, with AlphaSim corrected. MTG, middle temporal gyrus; PosCG, postcentral gyrus; MFG, middle frontal gyrus; FG, fusiform gyrus; IPL, inferior parietal lobule; PreCG, precentral gyrus. The left hemisphere is on the left. 
TABLE 3 | Brain areas showing significant differences in frequency specific FCD between patients with GAD and HCs.

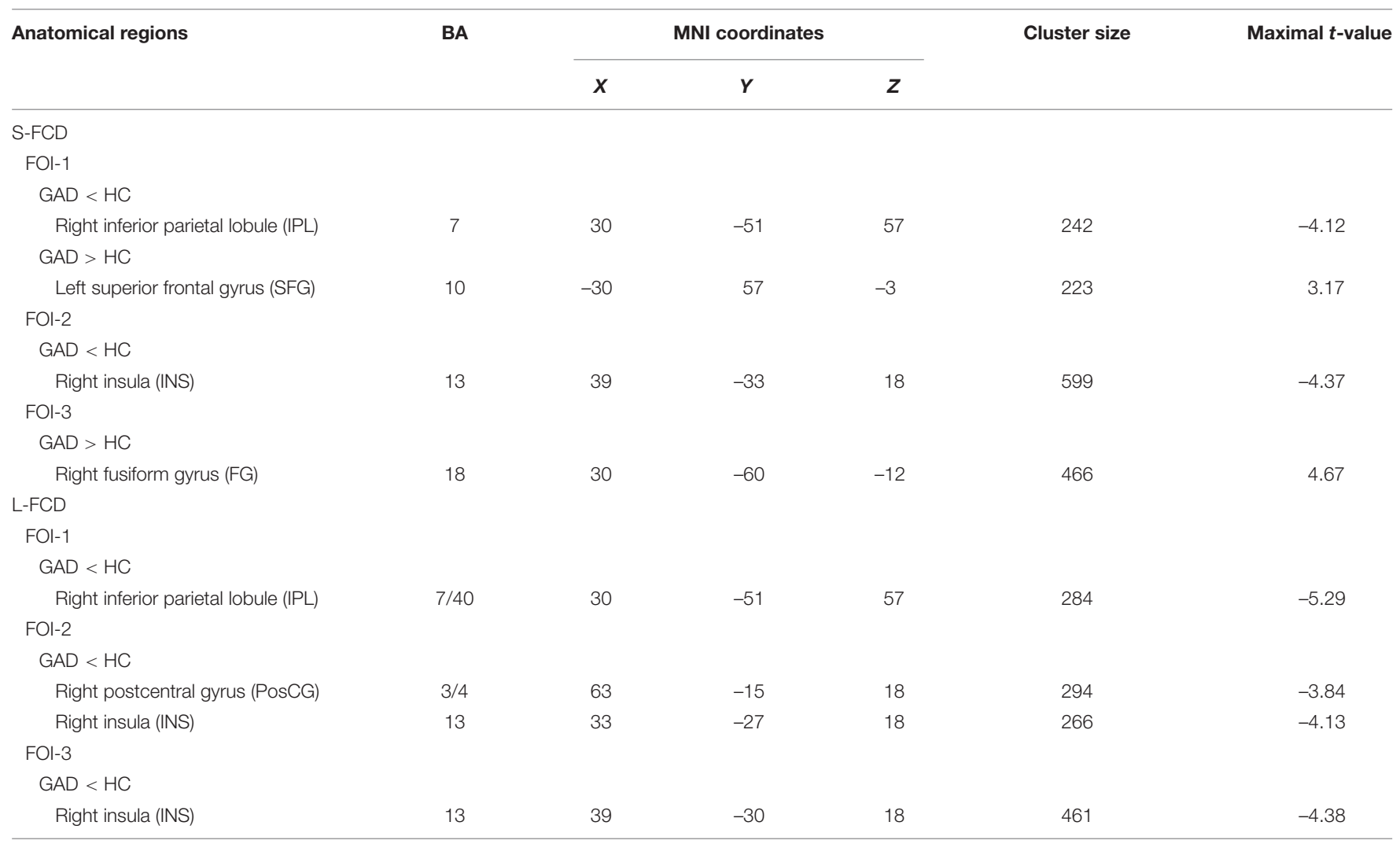

Maximal $t$-value, statistical value of peak voxel showing S-FCD/L-FCD differences between two groups. BA, Brod mann's area; MNI, Montreal Neurological Institute. $X Y / Z$, coordinates of peak locations in the MNI space. $P<0.05$, corrected for AlphaSim.

\section{Correlation Analyses}

As seen in Figure 5, within FOI-N, state anxiety inventory (SAI) score was positively correlated with L-FCD of the left FG in GAD $(r=0.43, P=0.016$, FDR corrected $)$. We also computed the correlation, respectively, between clinical scales and S-FCD/L-FCD in all frequency-specific regions that showed significant between-group differences, but no significant correlation was found $(P>0.05)$.

\section{Classification Performance}

The mean values of frequency-specific S-FCD and L-FCD with between-group difference within each cluster were extracted for discriminating GAD patients from HCs. We observed the classification performance when considering the $\mathrm{S}-\mathrm{FCD} / \mathrm{L}-\mathrm{FCD}$ of between-group difference region at each frequency band and the classification accuracy peaked when using the right INS of FOI-2 (74.65\%) (see Figure 6). The detailed classification performance of each region in each frequency band was presented in Supplementary Figures and Tables.

The classification performance of using discrepant S-FCD and L-FCD from FOI-N, FOI-S, and the combination of FOI-N and FOI-S were further examined. Results of S-FCD separately received the AUC values of $0.6117,0.7503$, and 0.9002 with corresponding classification accuracies of 72.94, 74.61, and $83.43 \%$ (Table 4 and Figure 7A). Compared to
S-FCD, the L-FCD showed better classification performance which separately achieved the AUC values of $0.7626,0.8547$, and 0.8633 with corresponding accuracies of $81.42,83.11$, and 84.53\%, respectively (Table 4 and Figure 7B). The classification performance were further improved by combining both S-FCD and L-FCD, as the AUC values of $0.8731,0.8993$, and 0.9414 were, respectively, obtained with corresponding classification accuracies of 84.75, 85.74, and 89.83\% (Table 4 and Figure 7C).

\section{DISCUSSION}

In the current study, frequency-specific FCD analysis was carried out to explore aberrant SFBO in adolescent patients with GAD. Our findings showed that GAD patients had significant differences in S-FCD and L-FCD across different frequency bands compared with HCs. Furthermore, significantly positive correlation between the SAI and L-FCD in the left FG was found in the conventional frequency band FOI-N $(0.01-0.08 \mathrm{~Hz})$. More importantly, the right insula was found to have the highest classification accuracy in the lower frequency band FOI-2 $(0.02-0.036 \mathrm{~Hz})$ in discriminating GAD patients from control participants compared with all other between-group difference regions and a satisfactory classification performance was achieved by combining the discrepant S-FCD and L-FCD values in all frequency bands. Our findings indicated that abnormal FCD in 


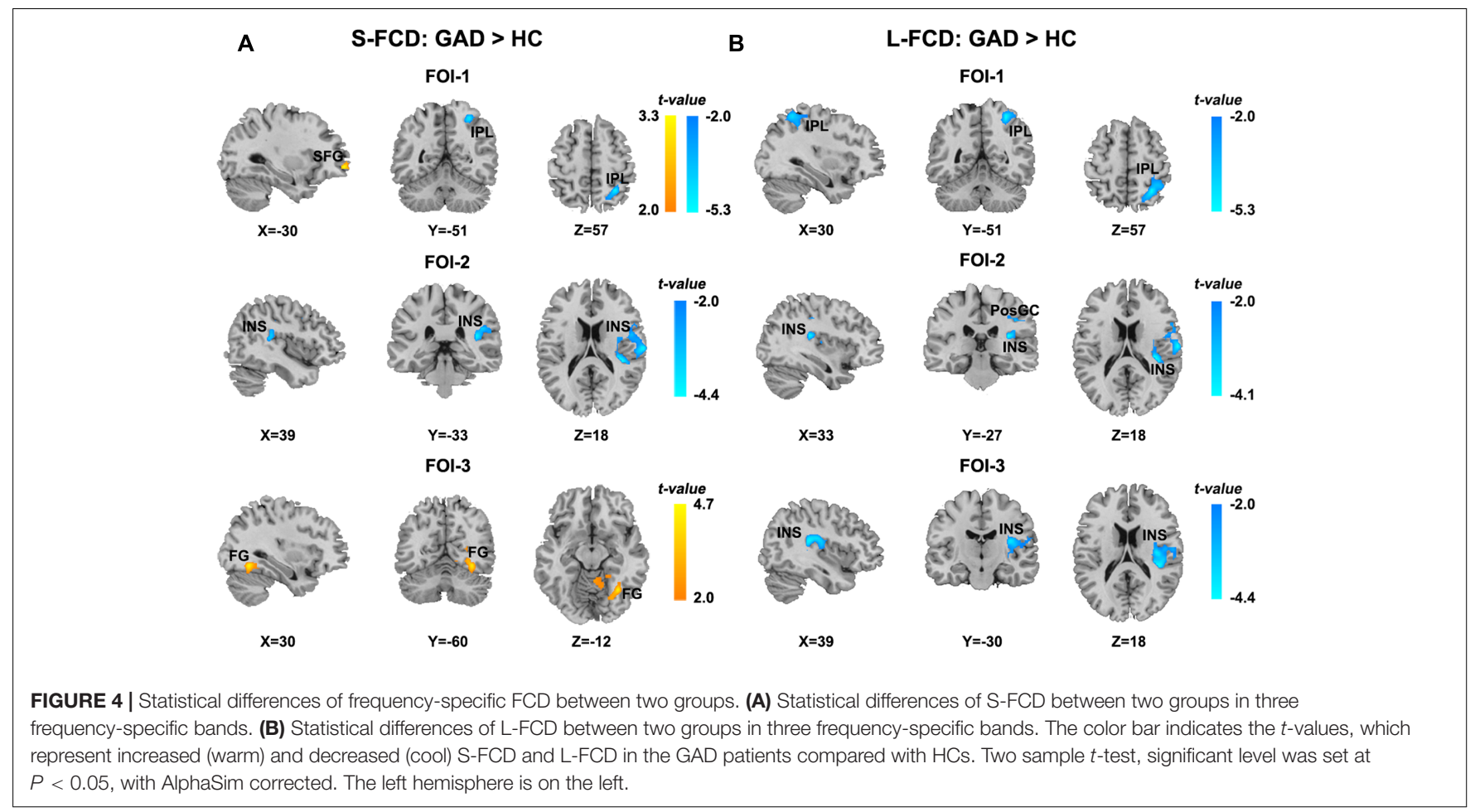

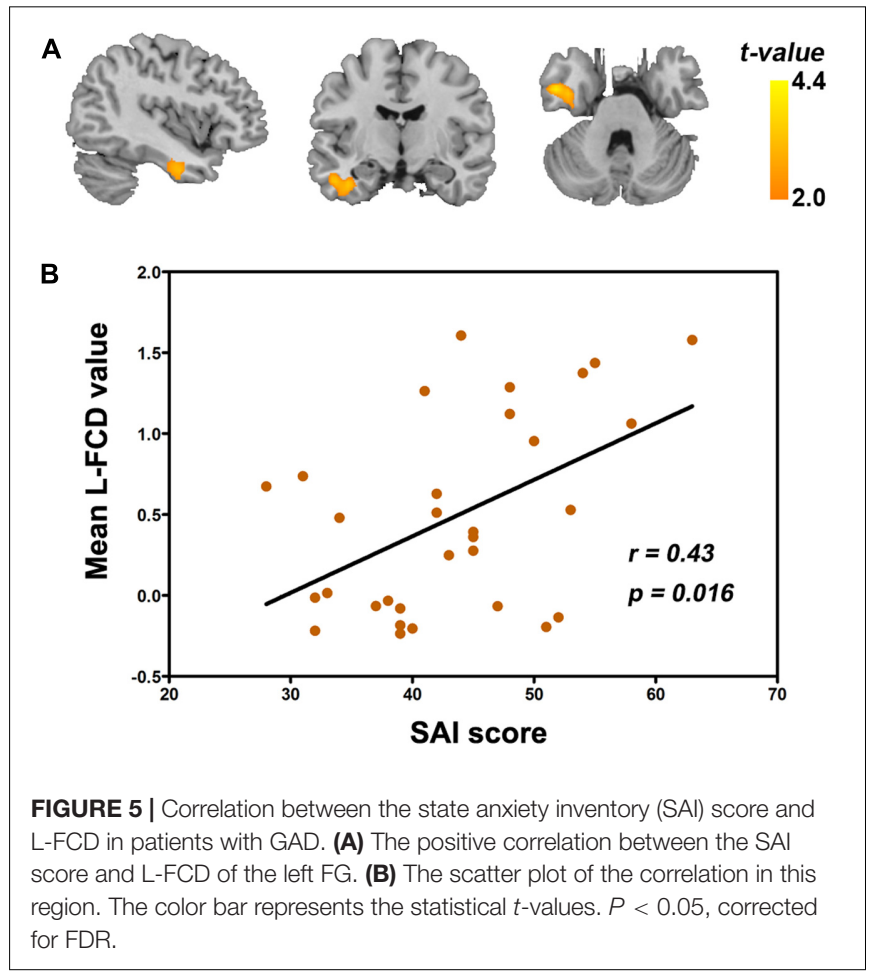

some brain regions in GAD patients was frequency dependent and frequency-specific FCD could be used in the diagnosis of an individual patient with GAD.

In the conventional frequency band FOI-N, brain regions with abnormal S-FCD in GAD patients were mainly located in the left MTG, left PosCG and left MFG, whereas brain regions with abnormal L-FCD were mainly located in the right PreCG, right IPL and left FG, showing disparately spatial patterns of the S-FCD versus L-FCD. The MTG is associated with recognition of known faces and accessing word meaning while reading (Acheson and Hagoort, 2013). Increased S-FCD in MTG may reflect that GAD patients show enhanced attention toward negative information in comparison to HCs. Moreover, the PreCG and PosCG, which have been reported to be involved in interoception processing (Northoff, 2013), displayed reduced S-FCD and L-FCD in GAD patients, respectively. A task-based fMRI study has depicted higher activities in PreCG in response to the anxiety-inducing words in GAD patients (Moon et al., 2015). Our results indicates disruption of FCD in PreCG and PosCG may induce abnormality in integration of network and mutual adjustment between cognitive function in most cases (Di Bono et al., 2017). Additionally, abnormal FCD in the MFG, IPL and FG were also found, and these regions have been involved in various of cognitive processes (Yang and Raine, 2009). Longterm existence of excessive and uncontrollable fear and worry in GAD patients might be thought to result in disruption of intrinsic cognitive function organization. Different from previous amygdala-based FC studies, our results showed that widespread changes of SFBO existed in adolescent GAD.

Notably, the GAD patients showed significantly positive correlation between increased L-FCD in the left FG and the SAI score that evaluates a kind of transient emotional state provoked by risky stimulus, including individual stress, worry, anxiety, distress, and excessive excitement of the autonomic nerve system (Spielberger et al., 1970). The FG, part of the temporal lobe and occipital lobe, has been indicated to serve a 


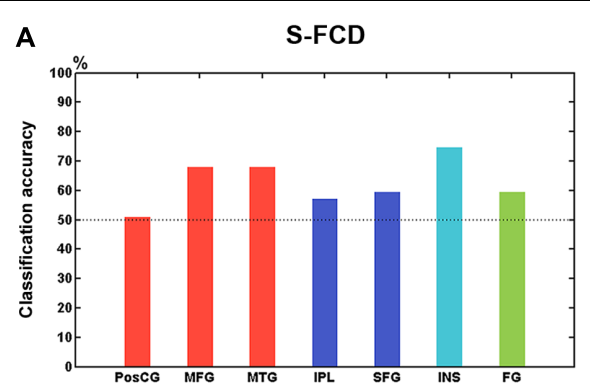

C

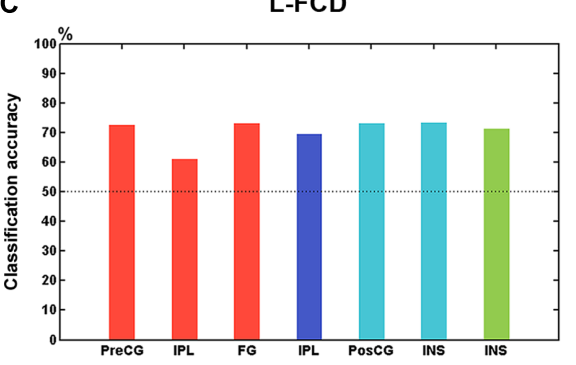

B

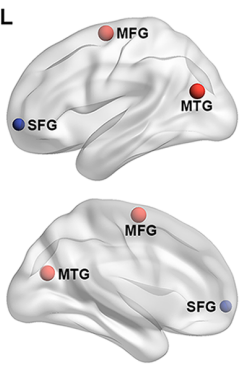

D

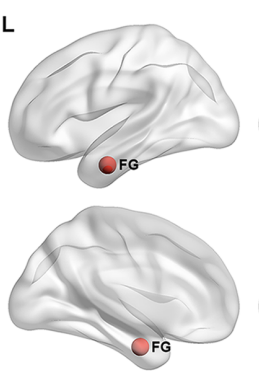

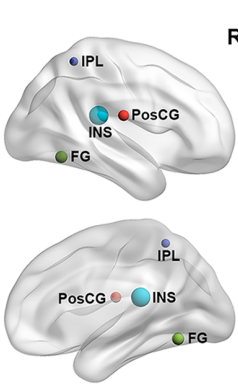

$\mathbf{R}$
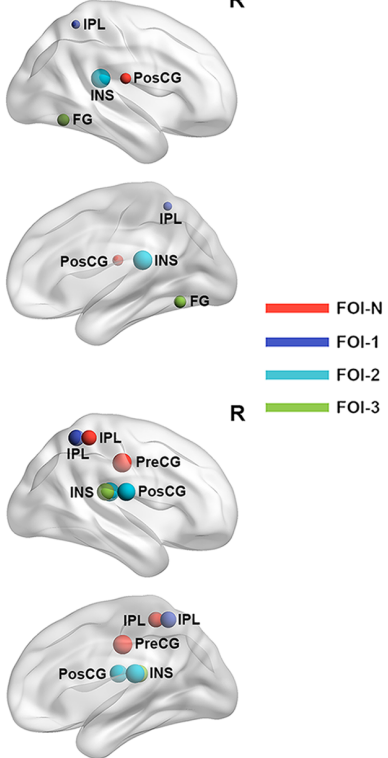

FIGURE 6 | Classification performance of each between-group difference region. The classification accuracy of each between-group difference region in S-FCD (A) and L-FCD (C). Rendering plot of the between-group difference regions in S-FCD (B) and L-FCD (D) used in the classification. Colors assigned in the sequence of red, blue, cyan, and green represent the frequency bands such as FOI-N, FOI-1, FOI-2, and FOI-3, respectively. The size of the node in (A,B) represents the accuracy of the normalized region classification performance. The left hemisphere is on the left.

vital function in recognizing face, body, and words (Haxby et al., 2002; Grill-Spector and Malach, 2004; Martin, 2007). Previous studies reported that the anxiety disorder patients possessed of abnormal activities in the FG when participating in the task related to emotional face, suggesting that FG might play an important role in emotion and visual processing (Gentili et al., 2008; Syal et al., 2012). Furthermore, resting-state fMRI study on social anxiety disorder patients also showed significantly increased FCstrength in the fusiform gyrus compared to HCs (Liu et al., 2015b). Specially, FG is usually functional connected with the hippocampus which belongs to the limbic system and it involves fear generalization processing (Xu and Sudhof, 2013). In

TABLE 4 | Discriminating the GAD participants from the HCs by receiver operating characteristic $(\mathrm{ROC})$ analyses.

\begin{tabular}{lcccc}
\hline Brain regions & AUC & Accuracy (\%) & Sensitivity (\%) & Specificity (\%) \\
\hline S-FCD & & & & \\
FOI-N & 0.6617 & 72.94 & 80.72 & 64.37 \\
FOI-S & 0.7503 & 74.61 & 71.03 & 78.60 \\
FOI-N + FOI-S & 0.9002 & 83.43 & 87.11 & 79.55 \\
L-FCD & & & & \\
FOI-N & 0.7626 & 81.42 & 90.71 & 71.98 \\
FOI-S & 0.8547 & 83.11 & 83.90 & 82.25 \\
FOI-N + FOI-S & 0.8633 & 84.53 & 83.44 & 85.73 \\
S-FCD + L-FCD & & & & 82.17 \\
FOI-N & 0.8731 & 84.75 & 87.13 & 89.11 \\
FOI-S & 0.8993 & 85.74 & 82.47 & 92.92 \\
FOI-N + FOI-S & 0.9414 & 89.83 & 87.15 &
\end{tabular}

a recent resting-state $\mathrm{FC}$ study in $\mathrm{GAD}$, the researchers reported that the increased FC between hippocampus and FG indicated that abnormal SFBO was mainly related to fear generalization associated with neural circuit and was responsible for the emotion dysregulation in GAD (Cui et al., 2016). In our study, the increased L-FCD in the left FG might be associated with the dysfunction of transient emotional processing, which might help to improve the emotional regulation abilities of adolescent GAD patients.

In three frequency-specific bands, GAD patients showed altered S-FCD and L-FCD primarily distributed over the right IPL, left SFG, right INS, right FG, and right PosCG, reflecting incomplete consistent inter-group difference regions compared with FOI-N. Both IPL and INS belong to the default mode network (DMN), which is the most well-characterized intrinsic connectivity network and its disruption can result in various emotional dysfunctions (Akansha et al., 2016). GAD patients always show deficiency in emotion processing such as limited self-control, concentrating difficulty, and response inhibition (Fonzo and Etkin, 2016). Our findings are in line with the perspective that emotional dysregulation derived from disrupted DMN is an universal phenomenon in GAD (Mochcovitch et al., 2014). Interestingly, both S-FCD and L-FCD in GAD patients were reduced in the right IPL at higher frequency band FOI-1 but showed no significant alterations at lower frequency bands such as FOI-2 and FOI-3, and reduced in the right INS at lower frequency bands FOI-2 but did not significantly change in FOI-1, which indicated that the alterations of FCD were frequency dependence in GAD patients and the frequency band FOI-1might be more sensitive to detect abnormal SFBO in right 

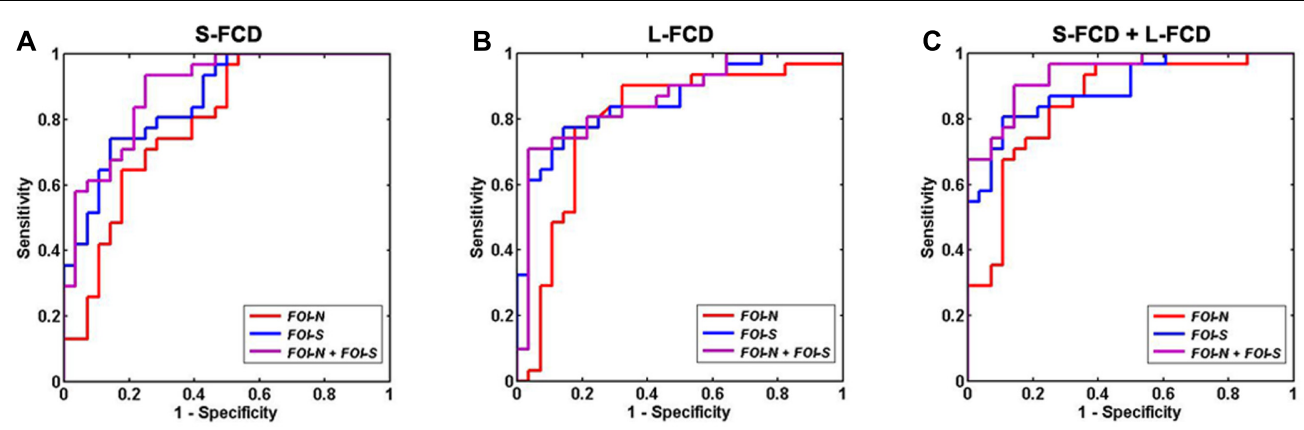

FIGURE 7 | Receiver operating characteristic (ROC) of discriminating GAD patients from HCs by using FCD values in the significant group difference regions. (A) S-FCD values in the significantly different regions; (B) L-FCD values in the significantly different regions; (C) a combination of S-FCD and L-FCD values in the significantly different regions. In (A-C), red, blue, and purple line represent the FCD in FOI-N, FOI-S, and FOI-N+FOI-S, respectively.

IPL than others, whereas the frequency band FOI-2 might be more sensitive to detect abnormal SFBO in right INS than others. Previous studies have observed frequency dependent changes of intrinsic brain fluctuations in resting-state in other diseases, suggesting that intrinsic brain activities are sensitive to specific frequency bands. For example, a resting-state study in social anxiety disorder has showed that ALFF reduced in the $0.01-0.027 \mathrm{~Hz}$ but did not change in the $0.027-0.073 \mathrm{~Hz}$ (Zhang et al., 2015). Another study suggested that the alterations of low-frequency oscillation amplitudes in specific brain regions in major depressive disorder patients could be more sensitively detected in the $0.01-0.027 \mathrm{~Hz}$ rather than the $0.027-0.073 \mathrm{~Hz}$ bands (Wang L. et al., 2016). Since brain fluctuations of different frequencies may have different physiological implications, the GAD-related abnormal FCD in distinct brain regions may have different sensitivity toward different frequency bands. Moreover, increased S-FCD in the left SFG (FOI-1) and in the right FG (FOI-3), and reduced L-FCD in the right PosCG (FOI-2) in GAD patients were also found in the present work. These findings also suggested that widespread brain regions were presented in frequency-specific disruptions and diverse sensitivity in detecting alterations of SFBO might rely on choosing frequency bands in adolescent $\mathrm{GAD}$ patients.

We further observed the classification performance of the FCD value in sole between-group difference region when discriminating GAD patients from $\mathrm{HCs}$. We found that the insula in a lower frequency band FOI-2 had the optimal classification performance with the highest classification accuracy reaching $74.65 \%$, which was an acceptable accuracy for established diagnostic indicators (Swets, 1988). As mentioned above, decreased FCD in INS might reflected a disruption DMN in emotional dysregulation for GAD patients. Additionally, evidence from other perspective also demonstrated abnormal activity in INS during emotion regulation process in GAD patients. For example, task-based fMRI studies have shown the hyperactivity of insula in response to negative emotion in anxious individuals (Hoehn-Saric et al., 2004; Gentili et al., 2008; Etkin et al., 2011) and decreased activity when using anxious words stimuli after seven weeks of citalopram treatment (Paulus and Stein, 2010), which indicates that insula activity may be a key neural mechanism underlying anxiety-related processes. The insula is also thought to enable to perceive the information of salience, emotion, as well as attention and then integrate and send such information to amygdala (Nieuwenhuys, 2012; Baur et al., 2013), which has been found to be structurally connected to the insula [60]. Recent studies suggest that these processes are disrupted in adolescent patients with GAD who exhibited abnormal FC between the amygdala and the insula both in resting-state (Etkin et al., 2009; Roy et al., 2013; Hamm et al., 2014) and task-state (Hoehn-Saric et al., 2004; Moon et al., 2015), which possibly leads to emotional dysregulation due to a neglect of some typical information in interoceptive stimuli from potentially aversive body signals (Paulus and Stein, 2010). GAD adolescents often have uncontrollable fear and worry, possibly because the reduced insula FCD disrupted the function in integrating information transferred from the insula to the amygdala. Moreover, Mcclure et al. (2007) discovered a positive correlation between the scores in a state of anxiety and amygdala-insula FC, which was considered to be a biological marker of anxiety. Our results were in line with the previous findings that abnormal insula activity was ubiquitous in GAD and suggested that the specific symptomatology of GAD might be related to the abnormal insula activity in the lower frequency band $(0.02-0.036 \mathrm{~Hz})$. These also implied that the FCD in the insula in the lower frequency band could be as a potential biomarker to discriminate adolescent patients with GAD from HCs. Further study should pay more attention to investigate the pathophysiological mechanisms of insula in GAD.

In addition, the ROC results indicated that the L-FCD has better classification performance for discriminating the GAD patients from the HCs than S-FCD when combining group difference regions in three different frequency band conditions, reflecting that alteration of long-range functional connections is a more obvious biomarker in GAD. Previous study reported that only aberrant L-FCD was found in post-traumatic stress disorder (PSD) (Zhang et al., 2016), indicating neurodegenerative disruption of intrinsic oscillations and connectivity in PSD mainly affected long-distance connections (Liu et al., 2014). Our results showed that frequent worry in GAD patients might more result from the disruptions in long-distance connections 
which were associated with the consequent loss of brain network efficiency. Finally, the combination of S-FCD and L-FCD in all frequency-specific difference regions was used to discriminate GAD patients from $\mathrm{HCs}$ and the optimum classification performance achieved that the AUC value was 0.9414 and its corresponding classification accuracy was $89.83 \%$. Recently, a multimodal machine learning study on separating GAD and major depression from HCs achieved 90.10\% accuracy by combining the data in clinical, cortisol, and structural MRI (Hilbert et al., 2017). To our knowledge, there is few study discriminating GAD patients from $\mathrm{HCs}$ adopting resting-state FC approach, but results from a research on social anxiety disorder by using whole-brain FC have showed a correct classification rate of $82.5 \%$ (Liu et al., 2015a). Compared with previous studies, our frequency-specific FCD approach had a satisfactory classification performance and we thus inferred that frequencyspecific S-FCD and L-FCD could be employed as an effective means to diagnose GAD.

However, several limitations were noteworthy. First, the sample data was limited in the current study, so large sample is needed in future studies. Second, we used a relatively low sampling rate ( $\mathrm{TR}=3000 \mathrm{~ms}$ ) for multiple (36 slices) acquisitions in our study. Although this sampling rate helped to minimize some physiological noises such as cardiac fluctuations and respiratory, physiological noises could not be removed entirely. Thus, future research should try to apply a more rigorous data analysis method to remove noises. Third, we observed the SFBO in a low frequency band $(<0.08 \mathrm{~Hz})$ but ignored the high frequency band $(>0.08 \mathrm{~Hz}$ ) that is always thought to include some respiratory signals at the frequency range from 0.1 to $0.5 \mathrm{~Hz}$ (Cordes et al., 2001). Recently, several studies extended the observation of the pattern of intrinsic brain activity to a high frequency band $(>0.1 \mathrm{~Hz})$, which demonstrated consistent patterns with low frequency fluctuations $(<0.1 \mathrm{~Hz})$ (Boubela et al., 2013), indicating that some valuable physiological information might be included in the high frequency band. Finally, we only selected a single threshold to calculate FCD maps, so a range of thresholds can be tried to test the stability of the results in future study.

\section{CONCLUSION}

We used the frequency-specific FCD mappings to investigate the SFBO of the adolescent GAD patients. The results showed that GAD patients exhibited abnormal S-FCD and L-FCD

\section{REFERENCES}

Acheson, D. J., and Hagoort, P. (2013). Stimulating the brain's language network: syntactic ambiguity resolution after TMS to the inferior frontal gyrus and middle temporal gyrus. J. Cogn. Neurosci. 25, 1664-1677. doi: 10.1162/jocn_ a_00430

Akansha, M., Roberto, A. J., Abhishek, M., Aileen, L., Kathryn, J., and Carney, M. J. (2016). The significance of the default mode network (DMN) in neurological and neuropsychiatric disorders: a review. Yale J. Biol. Med. 89, 49-57.

Ashburner, J. (2007). A fast diffeomorphic image registration algorithm. Neuroimage 38, 95-113. doi: 10.1016/j.neuroimage.2007.07.007 in widespread brain regions across different frequency bands, and a satisfactory classification performance was gained in discriminating GAD patients from $\mathrm{HCs}$ by using abnormal frequency-specific FCD values. Thus, it seems possible that frequency-specific FCD can be used as an effective biomarker in diagnosing GAD in the future. The findings highlight the importance of frequency specificity in SFBO and will deepen our understanding of the pathophysiological mechanism underlying GAD.

\section{AUTHOR CONTRIBUTIONS}

$\mathrm{ZZ}$ and ML contributed equally to this work. ML and LL designed and performed the experiments. ZZ, YX, and ZY researched data, contributed to discussion, and wrote, reviewed, and edited the manuscript. $\mathrm{ML}, \mathrm{BH}$, and $\mathrm{WZ}$ researched data, contributed to discussion, and reviewed and edited the manuscript. TH, YZ, FY, YZ, LS, LL, JG, and DM contributed to discussion, and reviewed and edited the manuscript.

\section{FUNDING}

This work was supported by the National Basic Research Program of China (973 Program) (Nos. 2013CB835100 and 2014CB744600); the National Science and Technologic Program of China (No. 2015BAI13B02); the National Natural Science Foundation of China (Nos. 61210010, 61632014, 81601180, and 81671353); the Program of International S\&T Cooperation of MOST (No. 2013DFA11140); and the Program of Beijing Municipal Science \& Technology Commission (No. Z171100000117005).

\section{ACKNOWLEDGMENTS}

We would like to thank all participants in this study. We also thank Lingshan Huang for English language help.

\section{SUPPLEMENTARY MATERIAL}

The Supplementary Material for this article can be found online at: https://www.frontiersin.org/articles/10.3389/fnhum. 2017.00549/full\#supplementary-material

Athanasiou, A., Klados, M. A., Styliadis, C., Foroglou, N., Polyzoidis, K., and Bamidis, P. D. (2016). Investigating the role of alpha and beta rhythms in functional motor networks. Neuroscience doi: 10.1016/j.neuroscience.2016.05. 044 [Epub ahead of print].

Baur, V., Hanggi, J., Langer, N., and Jancke, L. (2013). Resting-state functional and structural connectivity within an insula-amygdala route specifically index state and trait anxiety. Biol. Psychiatry 73, 85-92. doi: 10.1016/j.biopsych.2012.06.003

Beck, A. T., and Beamesderfer, A. (1974). Assessment of depression: the depression inventory. Mod. Probl. Pharmacopsychiatry 7, 151-169. doi: 10.1159/000395074

Beesdo, K., Pine, D. S., Lieb, R., and Wittchen, H. U. (2010). Incidence and risk patterns of anxiety and depressive disorders and categorization of 
generalized anxiety disorder. Arch. Gen. Psychiatry 67, 47-57. doi: 10.1001/ archgenpsychiatry.2009.177

Blair, K. S., Geraci, M., Smith, B. W., Hollon, N., Devido, J., Otero, M., et al. (2012). Reduced dorsal anterior cingulate cortical activity during emotional regulation and top-down attentional control in generalized social phobia, generalized anxiety disorder, and comorbid generalized social phobia/generalized anxiety disorder. Biol. Psychiatry 72, 476-482. doi: 10.1016/j.biopsych.2012.04.013

Boubela, R. N., Kalcher, K., Huf, W., Kronnerwetter, C., Filzmoser, P., and Moser, E. (2013). Beyond noise: using temporal ICA to extract meaningful information from high-frequency fMRI signal fluctuations during rest. Front. Hum. Neurosci. 7:168. doi: 10.3389/fnhum.2013.00168

Buzsa, G., and Draguhn, A. (2004). Neuronal oscillations in cortical networks. Science 304, 1926-1929. doi: 10.1126/science.1099745

Chavira, D. A., Stein, M. B., Bailey, K., and Stein, M. T. (2004). Child anxiety in primary care: prevalent but untreated. Depress Anxiety 20, 155-164. doi: $10.1002 /$ da.20039

Colominas, M. A., Schlotthauer, G., and Torres, M. E. (2014). Improved complete ensemble EMD: a suitable tool for biomedical signal processing. Biomed. Signal Process. Control 14, 19-29. doi: 10.1016/j.bspc.2014.06.009

Cordes, D., Haughton, V. M., Arfanakis, K., Carew, J. D., Turski, P. A., and Moritz, C. H. (2001). Frequencies contributing to functional connectivity in the cerebral cortex in "resting-state" data. AJNR Am. J. Neuroradiol. 22, 1326-1333.

Cortes, C., and Vapnik, V. (1995). Support-vector networks. Mach. Learn. 20, 273-297. doi: 10.1007/BF00994018

Cui, H., Zhang, J., Liu, Y., Li, Q., Li, H., Zhang, L., et al. (2016). Differential alterations of resting-state functional connectivity in generalized anxiety disorder and panic disorder. Hum. Brain Mapp. 37, 1459-1473. doi: 10.1002/ hbm.23113

Di Bono, M. G., Priftis, K., and Umilta, C. (2017). Bridging the gap between brain activity and cognition: beyond the different tales of fMRI data analysis. Front. Neurosci. 11:31.

Etkin, A., Egner, T., and Kalisch, R. (2011). Emotional processing in anterior cingulate and medial prefrontal cortex. Trends Cogn. Sci. 15, 85-93. doi: 10.1016/j.tics.2010.11.004

Etkin, A., Prater, K. E., Hoeft, F., Menon, V., and Schatzberg, A. F. (2010). Failure of anterior cingulate activation and connectivity with the amygdala during implicit regulation of emotional processing in generalized anxiety disorder. Am. J. Psychiatry 167, 545-554. doi: 10.1176/appi.ajp.2009.09070931

Etkin, A., Prater, K. E., Schatzberg, A. F., Menon, V., and Greicius, M. D. (2009). Disrupted amygdalar subregion functional connectivity and evidence of a compensatory network in generalized anxiety disorder. Arch. Gen. Psychiatry 66, 1361-1372. doi: 10.1001/archgenpsychiatry.2009.104

Farago, P., Tuka, B., Toth, E., Szabo, N., Kiraly, A., Csete, G., et al. (2017). Interictal brain activity differs in migraine with and without aura: resting state fMRI study. J. Headache Pain 18:8. doi: 10.1186/s10194-016-0716-8

Fonzo, G. A., and Etkin, A. (2016). Brain connectivity reflects mental and physical states in generalized anxiety disorder. Biol. Psychiatry 80, 733-735. doi: 10.1016/ j.biopsych.2016.08.026

Gentili, C., Gobbini, M. I., Ricciardi, E., Vanello, N., Pietrini, P., Haxby, J. V., et al. (2008). Differential modulation of neural activity throughout the distributed neural system for face perception in patients with Social Phobia and healthy subjects. Brain Res. Bull. 77, 286-292. doi: 10.1016/j.brainresbull.2008.08.003

Grill-Spector, K., and Malach, R. (2004). The human visual cortex. Annu. Rev. Neurosci. 27, 649-677. doi: 10.1146/annurev.neuro.27.070203.144220

Hamm, L. L., Jacobs, R. H., Johnson, M. W., Fitzgerald, D. A., Fitzgerald, K. D., and Langenecker, S. A. (2014). Aberrant amygdala functional connectivity at rest in pediatric anxiety disorders. Biol. Mood Anxiety Disord. 4, 15. doi: 10.1186/s13587-014-0015-4

Han, Y., Wang, J., Zhao, Z., Min, B., Lu, J., Li, K., et al. (2011). Frequencydependent changes in the amplitude of low-frequency fluctuations in amnestic mild cognitive impairment: a resting-state fMRI study. Neuroimage 55, 287-295. doi: 10.1016/j.neuroimage.2010.11.059

Haxby, J. V., Hoffman, E. A., and Gobbini, M. I. (2002). Human neural systems for face recognition and social communication. Biol. Psychiatry 51, 59-67. doi: 10.1016/S0006-3223(01)01330-0

Hilbert, K., Lueken, U., and Beesdo-Baum, K. (2014). Neural structures, functioning and connectivity in Generalized Anxiety Disorder and interaction with neuroendocrine systems: a systematic review. J. Affect. Disord. 158, 114-126. doi: 10.1016/j.jad.2014.01.022

Hilbert, K., Lueken, U., Muehlhan, M., and Beesdo-Baum, K. (2017). Separating generalized anxiety disorder from major depression using clinical, hormonal, and structural MRI data: a multimodal machine learning study. Brain Behav 7:e00633. doi: 10.1002/brb3.633

Hoehn-Saric, R., Schlund, M. W., and Wong, S. H. (2004). Effects of citalopram on worry and brain activation in patients with generalized anxiety disorder. Psychiatry Res. 131, 11-21. doi: 10.1016/j.pscychresns.2004.02.003

Hoffman, D. L., Dukes, E. M., and Wittchen, H. U. (2008). Human and economic burden of generalized anxiety disorder. Depress. Anxiety 25, 72-90. doi: 10.1002/da.20257

Hu, X., Chen, S., Huang, C. B., Qian, Y., and Yu, Y. (2017). Frequency-dependent changes in the amplitude of low-frequency fluctuations in patients with Wilson's disease: a resting-state fMRI study. Metab. Brain Dis. 32, 685-692. doi: 10.1007/s11011-016-9946-3

Huang, N. E., Shen, Z., Long, S. R., Wu, M. C., Shih, H. H., Zheng, Q., et al. (1998). The empirical mode decomposition and the Hilbert spectrum for nonlinear and non-stationary time series analysis. Proc. R. Soc. Lond. A 454, 903-995. doi: 10.1098/rspa.1998.0193

Kessler, R. C., Berglund, P., Demler, O., Jin, R., Merikangas, K. R., and Walters, E. E. (2005). Lifetime prevalence and age-of-onset distributions of DSM-IV disorders in the national comorbidity survey replication. Arch. Gen. Psychiatry 62, 593-602. doi: 10.1001/archpsyc.62.6.593

Knyazev, G. G. (2007). Motivation, emotion, and their inhibitory control mirrored in brain oscillations. Neurosci. Biobehav. Rev. 31, 377-395. doi: 10.1016/j. neubiorev.2006.10.004

Lauth, B., Arnkelsson, G. B., Magnússon, P., Skarphéðinsson, G., Ferrari, P., and Pétursson, H. (2010). Validity of K-SADS-PL (schedule for affective disorders and schizophrenia for school-age children-present and lifetime version) depression diagnoses in an adolescent clinical population. Nord. J. Psychiatry 64, 409. doi: 10.3109/08039481003777484

Liao, M., Yang, F., Zhang, Y., He, Z., Song, M., Jiang, T., et al. (2013). Childhood maltreatment is associated with larger left thalamic gray matter volume in adolescents with generalized anxiety disorder. PLOS ONE 8:e71898. doi: 10.1371/journal.pone.0071898

Liao, M., Yang, F., Zhang, Y., He, Z., Su, L., and Li, L. (2014). Lack of gender effects on gray matter volumes in adolescent generalized anxiety disorder. J. Affect. Disord. 155, 278-282. doi: 10.1016/j.jad.2013.10.049

Lin, X., Jia, X., Zang, Y. F., and Dong, G. (2015). Frequency-dependent changes in the amplitude of low-frequency fluctuations in internet gaming disorder. Front. Psychol. 6:1471. doi: 10.3389/fpsyg.2015.01471

Liu, F., Guo, W., Fouche, J. P., Wang, Y., Wang, W., Ding, J., et al. (2015a). Multivariate classification of social anxiety disorder using whole brain functional connectivity. Brain Struct. Funct. 220, 101-115. doi: 10.1007/s00429013-0641-4

Liu, F., Zhu, C., Wang, Y., Guo, W., Li, M., Wang, W., et al. (2015b). Disrupted cortical hubs in functional brain networks in social anxiety disorder. Clin. Neurophysiol. 126, 1711-1716. doi: 10.1016/j.clinph.2014.11.014

Liu, W. J., Yin, D. Z., Cheng, W. H., Fan, M. X., You, M. N., Men, W. W., et al. (2015c). Abnormal functional connectivity of the amygdala-based network in resting-state FMRI in adolescents with generalized anxiety disorder. Med. Sci. Monit. 21, 459-467.

Liu, Y., Yu, C., Zhang, X., Liu, J., Duan, Y., Alexander-Bloch, A. F., et al. (2014). Impaired long distance functional connectivity and weighted network architecture in Alzheimer's disease. Cereb. Cortex 24, 1422-1435. doi: 10.1093/ cercor/bhs410

Lu, F. M., Zhou, J. S., Wang, X. P., Xiang, Y. T., and Yuan, Z. (2017). Shortand long-range functional connectivity density alterations in adolescents with pure conduct disorder at resting-state. Neuroscience 351, 96-107. doi: 10.1016/ j.neuroscience.2017.03.040

Makovac, E., Watson, D. R., Meeten, F., Garfinkel, S. N., Cercignani, M., Critchley, H. D., et al. (2016). Amygdala functional connectivity as a longitudinal biomarker of symptom changes in generalized anxiety. Soc. Cogn. Affect. Neurosci. 11, 1719-1728. doi: 10.1093/scan/nsw091

Martin, A. (2007). The representation of object concepts in the brain. Annu. Rev. Psychol. 58, 25-45. doi: 10.1146/annurev.psych.57.102904.190143 
Mcclure, E. B., Monk, C. S., Nelson, E. E., Parrish, J. M., Adler, A., and Blair, R. J. (2007). Abnormal attention modulation of fear circuit function in pediatric generalized anxiety disorder. Arch. Gen. Psychiatry 64, 97-106. doi: 10.1001/ archpsyc.64.1.97

Meyer, T. J., Miller, M. L., Metzger, R. L., and Borkovec, T. D. (1990). Development and validation of the Penn state worry questionnaire. Behav. Res. Ther. 28, 487-495. doi: 10.1016/0005-7967(90)90135-6

Mochcovitch, M. D., Da Rocha Freire, R. C., Garcia, R. F., and Nardi, A. E. (2014). A systematic review of fMRI studies in generalized anxiety disorder: evaluating its neural and cognitive basis. J. Affect. Disord. 167, 336-342. doi: 10.1016/j.jad. 2014.06.041

Monk, C. S., Telzer, E. H., Mogg, K., Bradley, B. P., Mai, X., and Louro, H. M. (2008). Amygdala and ventrolateral prefrontal cortex activation to masked angry faces in children and adolescents with generalized anxiety disorder. Arch. Gen. Psychiatry 65, 568-576. doi: 10.1001/archpsyc.65.5.568

Moon, C. M., Yang, J. C., and Jeong, G. W. (2015). Explicit verbal memory impairments associated with brain functional deficits and morphological alterations in patients with generalized anxiety disorder. J. Affect. Disord. 186, 328-336. doi: 10.1016/j.jad.2015.07.038

Nieuwenhuys, R. (2012). The insular cortex: a review. Prog. Brain Res. 195, 123-163. doi: 10.1016/B978-0-444-53860-4.00007-6

Northoff, G. (2013). Unlocking the Brain: Coding, Vol. 1. New York, NY: Oxford University Press.

Paulus, M. P., and Stein, M. B. (2010). Interoception in anxiety and depression. Brain Struct. Funct. 214, 451-463. doi: 10.1007/s00429-010-0258-9

Pereira, F., Mitchell, T., and Botvinick, M. (2009). Machine learning classifiers and fMRI: a tutorial overview. Neuroimage 45, S199-S209. doi: 10.1016/j. neuroimage.2008.11.007

Qian, L., Zhang, Y., Zheng, L., Fu, X., Liu, W., Shang, Y., et al. (2016). Frequency specific brain networks in Parkinson's disease and comorbid depression. Brain Imaging Behav. 11, 224-239. doi: 10.1007/s11682-016-9514-9

Qin, W., Xuan, Y., Liu, Y., Jiang, T., and Yu, C. (2015). Functional connectivity density in congenitally and late blind subjects. Cereb. Cortex 25, 2507-2516. doi: 10.1093/cercor/bhu051

Roy, A. K., Fudge, J. L., Kelly, C., Perry, J. S., Daniele, T., Carlisi, C., et al. (2013). Intrinsic functional connectivity of amygdala-based networks in adolescent generalized anxiety disorder. J. Am. Acad. Child Adolesc. Psychiatry 52, 290.e2-299.e2. doi: 10.1016/j.jaac.2012.12.010

Shokri-Kojori, E., Tomasi, D., Wiers, C. E., Wang, G. J., and Volkow, N. D. (2016). Alcohol affects brain functional connectivity and its coupling with behavior: greater effects in male heavy drinkers. Mol. Psychiatry 22, 1185-1195. doi: $10.1038 / \mathrm{mp} .2016 .25$

Song, X. P., Zhang, Y., and Liu, Y. J. (2014). Frequency specificity of regional homogeneity in the resting-state human brain. PLOS ONE 9:e86818. doi: 10.1371 /journal.pone. 0086818

Spielberger, C. D., Gorsuch, R. L., and Lushene, R. E. (1970). Anxiety Inventory. Palo Alto, CA: Consulting Psychologists Press Inc.

Su, L., Wang, K., Fan, F., Su, Y., and Gao, X. (2008). Reliability and validity of the screen for child anxiety related emotional disorders (SCARED) in Chinese children. J. Anxiety Disord. 22, 612-621. doi: 10.1016/j.janxdis.2007.05.011

Swets, J. A. (1988). Measuring the accuracy of diagnostic systems. Science 240, 1285-1293. doi: 10.1126/science.3287615

Syal, S., Hattingh, C. J., Fouche, J. P., Spottiswoode, B., Carey, P. D., Lochner, C., et al. (2012). Grey matter abnormalities in social anxiety disorder: a pilot study. Metab. Brain Dis. 27, 299-309. doi: 10.1007/s11011-012-9299-5

Tomasi, D., Shokri-Kojori, E., and Volkow, N. D. (2016). Highresolution functional connectivity density: hub locations, sensitivity, specificity, reproducibility, and reliability. Cereb. Cortex 26, 3249-3259. doi: $10.1093 /$ cercor/bhv171

Tomasi, D., and Volkow, N. D. (2010). Functional connectivity density mapping. Proc. Natl. Acad. Sci. U.S.A. 107, 9885-9890. doi: 10.1073/pnas.1001414107

Tomasi, D., and Volkow, N. D. (2012). Aging and functional brain networks. Mol. Psychiatry 471, 549-558. doi: 10.1038/mp.2011.81

Wang, J., Zhang, Z., Ji, G. J., Xu, Q., Huang, Y., Wang, Z., et al. (2015). Frequencyspecific alterations of local synchronization in idiopathic generalized epilepsy. Medicine 94:e1374. doi: 10.1097/MD.0000000000001374

Wang, L., Kong, Q., Li, K., Su, Y., Zeng, Y., Zhang, Q., et al. (2016). Frequencydependent changes in amplitude of low-frequency oscillations in depression: a resting-state fMRI study. Neurosci. Lett. 614, 105-111. doi: 10.1016/j.neulet. 2016.01.012

Wang, W., Hou, J., Qian, S., Liu, K., Li, B., Li, M., et al. (2016). Aberrant regional neural fluctuations and functional connectivity in generalized anxiety disorder revealed by resting-state functional magnetic resonance imaging. Neurosci. Lett. 624, 78-84. doi: 10.1016/j.neulet.2016.05.005

Weng, Y., Qi, R., Liu, C., Ke, J., Xu, Q., Wang, F., et al. (2016). Disrupted functional connectivity density in irritable bowel syndrome patients. Brain Imaging Behav. doi: 10.1007/s11682-016-9653-z [Epub ahead of print].

Xie, H., and Wang, Z. (2006). Mean frequency derived via Hilbert-Huang transform with application to fatigue EMG signal analysis. Comput. Methods Programs 82, 114-120. doi: 10.1016/j.cmpb.2006.02.009

$\mathrm{Xu}$, W., and Sudhof, T. C. (2013). A neural circuit for memory specificity and generalization. Science 339, 1290-1295. doi: 10.1126/science.1229534

Yan, C. G., Cheung, B., Kelly, C., Colcombe, S., Craddock, R. C., Di Martino, A., et al. (2013). A comprehensive assessment of regional variation in the impact of head micromovements on functional connectomics. Neuroimage 76, 183-201. doi: 10.1016/j.neuroimage.2013.03.004

Yang, Y., and Raine, A. (2009). Prefrontal structural and functional brain imaging findings in antisocial, violent, and psychopathic individuals: a meta-analysis. Psychiatry Res. 174, 81-88. doi: 10.1016/j.pscychresns.2009.03.012

Zhang, Y., Xie, B., Chen, H., Li, M., Liu, F., and Chen, H. (2016). Abnormal functional connectivity density in post-traumatic stress disorder. Brain Topogr. 29, 405-411. doi: 10.1007/s10548-016-0472-8

Zhang, Y., Zhu, C., Chen, H., Duan, X., Lu, F., Li, M., et al. (2015). Frequencydependent alterations in the amplitude of low-frequency fluctuations in social anxiety disorder. J. Affect. Disord. 174, 329-335. doi: 10.1016/j.jad.2014. 12.001

Zhu, L., Li, Y., Wang, Y., Li, R., Zhang, Z., Lu, G., et al. (2016). Aberrant long-range functional connectivity density in generalized tonic-clonic seizures. Medicine 95:e3893. doi: 10.1097/MD.0000000000003893

Zuo, X. N., Di Martino, A., Kelly, C., Shehzad, Z. E., Gee, D. G., Klein, D. F., et al. (2010). The oscillating brain: complex and reliable. Neuroimage 49, 1432-1445. doi: 10.1016/j.neuroimage.2009.09.037

Conflict of Interest Statement: The authors declare that the research was conducted in the absence of any commercial or financial relationships that could be construed as a potential conflict of interest.

Copyright (c) 2017 Zhang, Liao, Yao, Hu, Xie, Zheng, Hu, Zhao, Yang, Zhang, Su, Li, Gutknecht and Majoe. This is an open-access article distributed under the terms of the Creative Commons Attribution License (CC BY). The use, distribution or reproduction in other forums is permitted, provided the original author(s) or licensor are credited and that the original publication in this journal is cited, in accordance with accepted academic practice. No use, distribution or reproduction is permitted which does not comply with these terms. 